\title{
Strategies and Practices of Computer-aided Business Translation
}

\author{
Aiping Tan \\ Foreign languages department of Sichuan Vocational and Technical College, Suining, 629000, \\ China
}

Keywords: computer-aided; translation technology; multiple needs

Abstract: With the overall improvement of China's national economy, new changes have taken place in the translation market. The more traditional methods of translation cannot meet the needs of people's diverse translation business in the current era. The service-based process can be divided into a number of stages, from the initial start-up phase to the further planning phase, to the specific implementation of the plan and the closing of the program. This paper elaborates the learning resources and functions of computer-assisted translation software for the readers' reference by expounding the diverse needs of the Chinese market for translation professionals.

\section{Introduction}

In our country, the translation market has been thriving in recent years. In general, the language-based service process can be divided into many stages, from the initial start-up phase, to the further planning phase, to the specific implementation of the plan and the closing of the program. In the start-up phase, it is mainly to obtain customer's relevant information, as well as some documents and information of the project; in the planning stage, it can further negotiate with customers for quotations and document analysis; during the implementation stage, it includes preprocessing, proofreading, real-time translation and quality. Many aspects of inspection and so on; the final is a concrete backup of the project document and summarize the reflection work.

\section{The Diversified Demand for Translation Professionals in the Chinese Market}

These steps in the translation work are more rigorous and meticulous as the translation market continues to expand, and language service companies need to pay attention to the candidate's understanding and expressiveness while recruiting talents, and focus on the use of various computer-aided software. Computer-assisted translation is different from pure computer translation. Computer-assisted translation is the process of translation memory and the combination of machine translation and manual proofreading. This is a set of human-computer interaction processes. It is very useful for non-literary text translation.

For translators, this means that they need to have a higher level of industry. The competition of current translators is not only a competition between translators and machines, but also a game between translators using computer-assisted translation techniques. An ideal translator should have 
solid basic qualities in translation, high-quality bilingualism, and relevant professional knowledge and flexible use of tools to assist these qualities. In short, an ideal translator should understand bilingualism, culture, and expertise. It can also be applied flexibly in translation technology.

\section{Kinds of computer-assisted translation tools and their influence}

\subsection{Classification of computer-assisted translation tools}

In general, computer-assisted translation tools can be divided into two categories. The first is common software and hardware. This includes the more commonly used word processing software, e-book software, and some search engines. Some hardware, such as rewritable optical drives, Digital cameras and some scanning instruments play a very important role in the processing of the language files before translation. At present, many of the original files of Fan Yu are in more formats. This is especially true for some picture format files. However, the software of computer-assisted translation tools currently cannot accurately recognize and edit the text between pictures. At this point, however, it can only be implemented with the help of OCR's software technology, and this is one of the important tools for translators to deal with pre-translation source files.

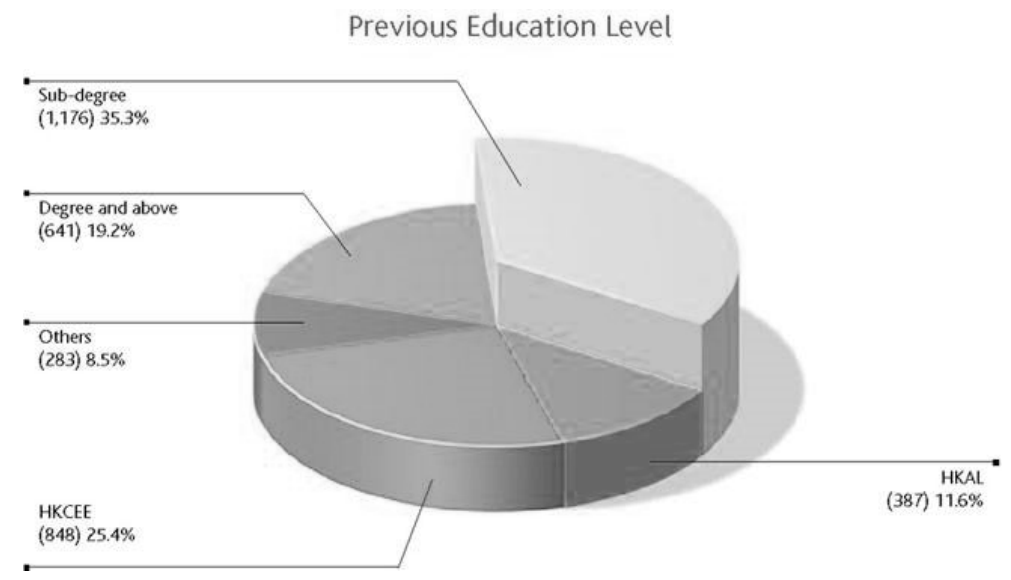

Fig. 1 Application statistics of computer-assisted translation in translator groups

The other is more professional translation software, which currently includes Yawing CAT, Snowman CAT, and Langtry CAT. In the process of translation, these software's can transfer some simple mechanical but repetitive work labor to the computer, and better assist the translation technicians in translation. At the same time, they can also help the work item to perform certain processing and analysis, and can be very effective. Help translators establish a clear and effective integration mechanism for translation and division of work, and achieve a smoother translation process.

\subsection{The Influence of Computer-Assisted Translation Technology on Translators}

In general, computer-aided translation technology focuses on how to skillfully use computer software to improve the efficiency of human translation, thereby ensuring the efficiency of human translation and ensuring the quality of further human translation. The core of such technology lies in the memory of translation. The memory of translation can record and store relevant content on a certain basis, which can significantly improve the efficiency of subsequent translation tasks. It can greatly help translators reduce work pressure and obtain a large amount of useful information in a short period of time. The structure of the computer-assisted translation technology system has 
certain advantages. For example, it can better improve the efficiency of translation, and it can effectively reduce unnecessary repetitive tasks for translators; at the same time, it can also ensure the translation of texts. Consistency of the meaning of the source text, in the overall translation, a source file through a number of people to cooperate, can be very effective to avoid inconsistent translation language.

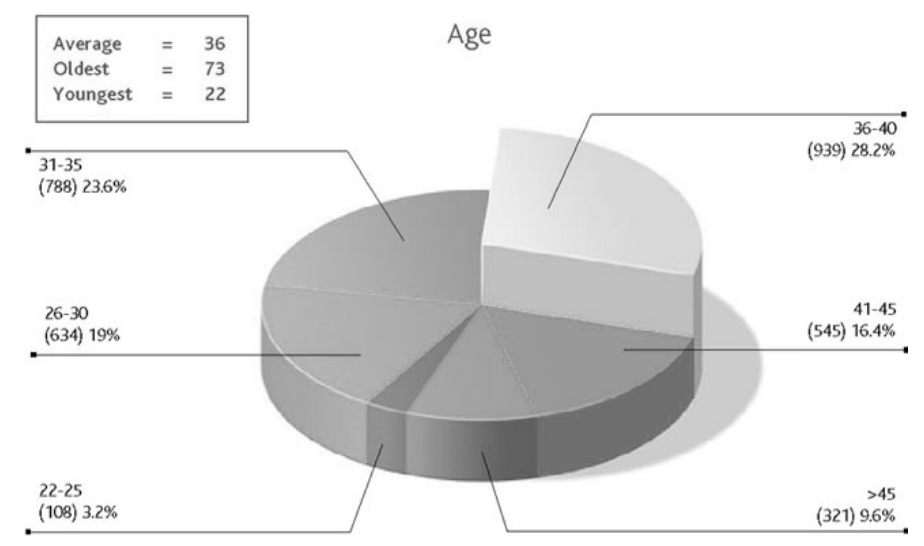

Fig.2 Age of Business Translators Using Computer Assisted Translation

At this time, the application of the terminology library can ensure the consistency of the translated text and the source text, thus ensuring the quality of the translation; on the other hand, the use of such a system structure can help the translator to greatly increase the efficiency of translation, thereby ensuring the overall translation of the Quality, but by reducing the cost of translation, memory data can also speed up the translation process, so that efficiency can be improved.

\section{Computer-assisted business translation practices}

\subsection{Learning Resources in Computer Aided Translation Tools}

In current mainstream computer-aided translation software, related manuals and video presentations are provided. These open learning resources are very helpful for translators to understand and learn. For example, the domestic translation group network provides a lot of learning experience for beginners, and an open platform for everyone to communicate with each other, these learning opportunities can be very effective to help translators understand the specific process of the language service in the information age and its research direction. .

\subsection{Translators' subjectivity in computer-assisted translation}

The working characteristics of computer-assisted translation and its own flaws determine that computer-assisted translation still cannot replace human translation. In the process of translation, the translator is still the main body of translation and plays a leading role in translation. According to scholars such as Cha Mingjian, the translator's subjectivity refers to the subjective initiative expressed by the translator in order to realize the purpose of translation, on the premise that the translator respects the target of the translation, and is embodied in the translator's cultural awareness, cultural character and culture, and aesthetic creativity. Conduct a consistency check on it to obtain:

$$
\mathrm{CR}=0.0282<0.1
$$

The translation strategy, macroscopically grasping the discourse, enhances the coherence and integrity of the translation. The core of such technology lies in the memory of translation. The memory of translation can record and store relevant content on a certain basis, which can 
significantly improve the efficiency of subsequent translation tasks.

\subsection{Computer-Assisted Translation in the Morning to Translators}

The subjectivity of translators in computer-assisted translation should extend through the entire process of translation. Specifically, translators should have forward-looking awareness before translation. We know that computer-assisted translation software does not help the translator itself. After the translation, the translator should co-ordinate the full text, macro-control the translation, grasp the intrinsic style and the external form of the translation as a whole, and pay attention to the relevance between the translation paragraphs, and try to avoid the mutation of the meaning and style, ensure the translation. Semantic coherence and consistency of styles improve the quality of translations.

\section{Summary}

The translation market has been flourishing in recent years in China. In general, the language-based service process can be divided into many stages, from the initial stage of startup to the further planning stage, to the specific implementation of the program, and to the finalization. The language-based service-based process can be divided into a number of stages, from the initial start-up phase, to the further planning stage, to the implementation of the program and the closing of the program. These steps are more rigorous and meticulous as the translation market continues to expand, and language service companies need to pay attention to the candidate's understanding and expressiveness while recruiting talents, and focus on the use of various computer-aided software. For translators, this means that they need to have a higher level of industry. Computer-assisted translation technology can skillfully use computer software to improve the efficiency of human translation, thus ensuring the efficiency of human translation and ensuring the quality of further human translation. It is a great improvement for translators' work efficiency.

\section{Acknowledgement}

2012 China Shippers Association foreign economy and trade"Pragmatic failure of pragmatic Strategies in Cross-cultural Business Communication from the Perspective of adaptation Theory (S-A-12028)"

\section{References}

[1] Qing Y U, Qin C F, Department B E. On translation strategies of local tourist publicity-orientated materials based on “Allusion Tracing”[J]. Journal of Anhui Technical College of Water Resources \& Hydroelectric Power, 2018.

[2] Liu L. Research on Principles and Strategies of Business English Translation based on Functional Perspective[C]// International Conference on Humanity, Education and Social Science. 2016.

[3] Rajesh M A, Toomey J E, Retime J O B. Developing a procurement path determination chart SEM-based approach[J]. Construction Management \& Economics, 2016, 33(11):1-21. 